\title{
Performance of ultrasound based measurement of 3D displacement using a curvilinear probe for organ motion tracking
}

Authors:

Emma J. Harris (corresponding author), Naomi R. Miller, Jeffrey C. Bamber, Philip

M. Evans and J. Richard N. Symonds-Tayler. Joint Department of Physics, Institute of Cancer Research, Sutton, Surrey, U.K.

Contact details:

Institute of Cancer Research, Joint Department of Physics, Cotswold road, Sutton, Surrey, SM2 5PT, U.K.

Tel: ++442086613478

Fax: ++44 2086433812

eharris@icr.ac.uk

Keywords: Curvilinear transducer, 3D speckle tracking, image guided radiotherapy, organ motion.

Abstract: Three-dimensional (3D) soft tissue tracking is of interest for monitoring organ motion during therapy. Our goal is to assess the tracking performance of a curvilinear 3D ultrasound probe in terms of the accuracy and precision of measured displacements. The first aim was to examine the depth dependence of the tracking performance. This is of interest because the spatial resolution varies with distance from the elevational focus and because the curvilinear geometry of the transducer 
causes the spatial sampling frequency to decrease with depth. Our second aim was to assess tracking performance as a function of the spatial sampling setting (low, medium or high sampling). These settings are incorporated onto 3D ultrasound machines to allow the user to control the trade-off between spatial sampling and temporal resolution. Volume images of a speckle-producing phantom were acquired before and after the probe had been moved by a known displacement (1,2 or $8 \mathrm{~mm})$. This allowed us to assess the optimum performance of the tracking algorithm, in the absence of motion. 3D speckle tracking was performed using 3D cross-correlation and sub-voxel displacements were estimated. The tracking performance was found to be best for $y$ displacements and poorest for $z$ displacements. In general, the performance decreased with depth, although the nature of the depth dependence was complex. Under certain conditions, the tracking performance was sufficient to be useful for monitoring organ motion. For example, at the highest sampling setting, for a $2 \mathrm{~mm}$ displacement, good accuracy and precision (an error and standard deviation of $<0.4 \mathrm{~mm}$ ) were observed at all depths and for all directions of displacement. The trade-off between spatial sampling, temporal resolution and size of field of view is discussed.

\section{Introduction}

The goal of our research is the real-time three-dimensional (3D) tracking of abdominal organ motion, which is currently the subject of much investigation within the fields of radiation therapy and HIFU therapy (Shimizu et al 2000, Pernot et al 2004, Webb 2006). Ultrasound is ideally suited for imaging tissue motion because of its real-time functionality and its soft tissue imaging capability. It has been used in 
radiation therapy to position patients prior to treatment and a number of different commercial 2D or 3D systems now exist (Chandra et al 2003, Cury et al 2006). Previously, we demonstrated the feasibility of imaging using B-mode ultrasound during radiotherapy (Hsu et al 2005). We showed that a 1D linear array transducer was able to measure the $2 \mathrm{D}$ displacement of a tissue phantom with sub-millimetre precision, regardless of electronic noise due to the operation of the radiotherapy equipment. The next steps are to develop the technique for 3D displacement estimation and to combine it with an absolute positioning system so that the measured motion of the internal anatomy can be placed in the treatment frame of reference. This paper deals with the first of these issues. It assesses the feasibility of 3D displacement estimation using a commercially available 3D abdominal ultrasound probe.

Previously, Morsy and von Ramm (1998) performed 3D correlation-based speckle tracking. To collect 3D volume-images, a piston transducer was moved in two dimensions across the surface of a stationary phantom. A small volume $(8 \mathrm{~mm} \times 8 \mathrm{~mm}$ $\times 8 \mathrm{~mm}$ ) was imaged by acquiring a radio-frequency $(\mathrm{RF})$ echo line at positions on a grid of $42 \times 42$ points. The RF data were envelope detected prior to speckle tracking. Correlation tracking of the phantom was demonstrated for small $(\sim 1 \mathrm{~mm})$ displacements in the axial and azimuthal directions by moving the phantom in between acquisitions. Chen et al (2005) demonstrated 3D correlation based speckle tracking using simulated RF data. They assessed the feasibility of using the technique to overcome decorrelation due to out-of-plane motion in 1D and 2D elasticity imaging. They showed that $3 \mathrm{D}$ correlation based tracking is more accurate than $2 \mathrm{D}$ tracking. They also introduced optimisation schemes to reduce the computational cost of 3D correlation based speckle tracking and discussed a hardware solution based on 
FPGAs (field programmable gate arrays) that would facilitate real-time tracking. Pernot et al (2004) proposed a method using four sub-apertures, each comprising seven elements, which were selected from a hemi-spherical phased array containing 200 elements. Each sub-aperture created a 1D beam of ultrasound that travelled in a unique direction. RF echo lines were acquired using each sub-aperture and 1D axial displacement was measured along the four directions using 1D RF speckle tracking. 3D displacements were then resolved from the four directional measurements. The technique was shown to be effective in monitoring tissue motion at a point a few millimetres in front of a HIFU heating zone and was successful in guiding HIFU treatment delivery to moving samples of in vitro bovine tissue. To date, the authors have only attempted to measure the motion of a small region of tissue $\left(<1 \mathrm{~cm}^{3}\right)$, as HIFU is a highly localised treatment. They have concentrated only on translational motion, although potential approaches for dealing with rotation are discussed.

Unlike the previous techniques, which used custom-built scanning equipment, our approach is to use a commercially available real-time 3D ultrasound scanner. Primarily, we are interested in tracking abdominal organs such as the liver, bladder, pancreas and prostate. To image these organs, we require a low frequency transducer with a large imaging depth. Commercially available probes that meet these criteria are curvilinear to generate a wide field of view at large imaging depths. 3D abdominal probes consist of a curvilinear transducer that is mechanically swept in a fan shape. A large field of view is particularly useful in the context of guiding therapy, as it facilitates patient set-up and would allow tracking of large tumours as well as adjacent organs that may be at risk. However, there are a number of potential disadvantages to using a curvilinear array for our purpose (displacement estimation). Firstly, the angle 
of incidence of the ultrasound beam changes with azimuthal and elevational position, which will cause the speckle pattern to decorrelate when there is movement in these directions. The effect of this decorrelation on tracking performance has not previously been studied, so the first aim of this paper was to determine whether displacement estimation with a 3D curvilinear probe is feasible given the angular decorrelation. A second potential disadvantage of curvilinear arrays is that due to the probe geometry, the spatial sampling in the azimuthal and elevational directions decreases with depth. In addition, as with all 1D ultrasound arrays, the elevational beam width decreases with distance from the elevational focus (which typically occurs at a depth of $\sim 6 \mathrm{~cm}$ ). Therefore, assuming that displacement estimation with a curvilinear probe is shown to be feasible, the second aim of this work was to characterise the accuracy and precision of displacement tracking as a function of imaging depth.

The third aim of this paper was to assess the tracking performance as a function of the spatial sampling setting on the scanner ("low", "medium" or "high"). This is different from the issue mentioned above of a depth-dependent sampling due to curvilinear geometry. Instead, we refer to the fact that manufacturers of 3D ultrasound scanners allow the user some degree of control over the trade-off between spatial sampling and acquisition speed via front panel settings. The temporal resolution for both mechanically swept 1D arrays and 2D array-based 3D ultrasound imaging systems is fundamentally limited by the speed of sound (Fenster et al 2001). The time taken to acquire a volume of data, $t_{v o l}$, is proportional to the product of the imaging depth (ID), the number of lines $(L)$, and the number of frames $(F)$. To track organ motion due to respiration in real-time, assuming a breathing cycle of 3-5 seconds, we require a volume acquisition rate, $1 / t_{\text {vol }}$, of $\sim 10 \mathrm{~Hz}$ (Keall et al 2004). To achieve this volume 
acquisition rate without restricting the field of view to a few cubic centimetres, spatial resolution has to be sacrificed using techniques such as parallel beam forming in receive mode (Smith et al 1991) or by reducing the spatial sampling frequency (so as to reduce $L$ or $F$ ). As mentioned above, the latter can be achieved using front panel settings. Therefore, it would be worthwhile investigating the effect of reduced spatial sampling on the accuracy and precision of displacement estimation.

If the organ is in motion during data acquisition, this will cause the speckle pattern to blur and decorrelate, which will reduce tracking performance (Pernot et al, 2004). The extent of decorrelation due to motion will depend on the speed, direction and variability of that motion. In this initial study, volume-images of a phantom were acquired before and after the transducer had been moved by a known displacement, a method that we refer to as "step and shoot". This allowed characterisation of the system under optimum conditions so that we could determine and understand the measurement errors in the absence of motion. A future paper will examine the additional degradation in tracking performance due to object motion during imaging.

\section{Methods and materials:}

\subsection{Experimental set-up}

A 3D curvilinear ultrasound probe with a broadband frequency of $\sim 4-7 \mathrm{MHz}$ (Model: 3D4-7EK, Medison Co., Ltd, Seoul, S. Korea) was used to image a phantom. The probe acquires a volumetric image by sweeping a $1 \mathrm{D}$ transducer array about an axis parallel to the transducer face whilst capturing $2 \mathrm{D}$ images at regular intervals so that

they form a fan shape. The scanner can record up to 50 volumes of B-mode data 
(Accuvix, Medison Co., Ltd, Seoul, S. Korea). These are stored in 8-bit binary format, the size of the matrix being defined in rows $\times$ columns $\times$ frames.

The probe was clamped to a mechanical articulated arm, which was mounted on a three-axis of motion translational stage (Nioutsikou et al. 2006). The position of the transducer was controlled by three stepper motors driven by commands from a PC. To assess tracking accuracy, we compared measured displacement vectors with known displacements. This required knowledge of any rotation between the coordinate system of the translational stage and that of the transducer. We attempted to attach the transducer to the translational stage such that visually, there was no rotation between the two systems. However, any residual misalignment was determined as described in Appendix A.

\subsection{Tissue phantom experiments}

A homogeneous phantom, approximately $20 \mathrm{~cm} \times 10 \mathrm{~cm} \times 8 \mathrm{~cm}$ in size and made of a water-based gel (14\% by weight porcine skin powdered gelatine) loaded with $2 \%$ by weight polyethylene scatterers, was used to simulate the ultrasound speckle pattern characteristic of homogeneous tissue, such as liver parenchyma. The speed of sound in the phantom was $\sim 1580 \mathrm{~m} / \mathrm{s}$. The phantom was placed at the bottom of a large tank of water on top of ultrasound absorbent material (a polyurethane rubber, Aptflex F28, Precision Acoustics, Dorset UK) to reduce the intensity of ultrasound reflections from the bottom surface of the tank. The phantom and absorbent material were fixed securely in place and remained stationary throughout the experiments. 
Rather than altering the position of the phantom, known displacements were achieved by moving the transducer using the translation stage. The transducer was moved over distances of 1,2 and 8mm in each of three independent directions ( $x, y$ and $z$ ), as well as in all three directions simultaneously. A minimum displacement of $1 \mathrm{~mm}$ was chosen as we do not require sub-millimetre accuracy for our application and a maximum displacement of $8 \mathrm{~mm}$ was chosen to represent a typical maximum instantaneous organ displacement (Balter et al 1995; Nederveen et al 2002). At each position, and for each of the three sampling settings (low, medium and high), up to 20 consecutive volume-images were acquired. The height of the phantom was $10 \mathrm{~cm}$. To obtain measurements at depths greater than $10 \mathrm{~cm}$, two experiments were performed, the first using an imaging depth of $13 \mathrm{~cm}$ and the second using an imaging depth of $20 \mathrm{~cm}$. In the first experiment, the front face of the probe was initially positioned at a height of approximately $3 \mathrm{~cm}$. This was changed to $10 \mathrm{~cm}$ for the second experiment.

The imaging (or probe) geometry is shown in figure 1 . For a point $P(x, y, z)$ in the Cartesian co-ordinate system, $x, y$ and $z$ are defined by

$$
\begin{aligned}
& x=(b+r) \sin \theta \\
& y=-D \cos \varphi \\
& z=D \sin \varphi \\
& \text { where } \\
& D=(b+r) \cos \theta-a)
\end{aligned}
$$

where $r$ is the axial distance of point $P$ from the face of the transducer, $\theta$ is the azimuthal angle, which is the angle between point $P$ and the central line of the frame, $\varphi$ is the sweep angle, which is the angle between the imaging plane containing $P$ and the central axis, $a$ is the distance between the physical pivot point of the ultrasound 
transducer and the virtual apex of the sector described by the ultrasound field and $b$ is the radius of curvature of the curvilinear transducer (see figure 1). The azimuthal angle and sweep angle were set to $69^{\circ}$ and $65^{\circ}$ respectively, for all experiments.

Assuming a perfectly aligned system, from equation set 1 , an $x$ motion within the Cartesian geometrical system produces an axial $(r)$ and azimuthal $(\theta)$ motion in the probe's geometrical system, a $z$ motion produces an axial $(r)$ and elevational $(\varphi)$ motion, and a $y$ motion produces a combination of all three motions.

A volume-image is a $3 \mathrm{D}$ matrix in which each element represents one image voxel. For fixed sweep and azimuthal angles, the size of the matrix is determined by the imaging depth (no. of rows $(R)$ ) and the sampling setting (no. of columns or lines $(L)$ and no. of frames $(F))$. The syntax $[R, L, F]$ will be used to denote the size of an image matrix or sub-regions thereof. Table 1 gives the number of rows and the axial sampling frequency (defined as the number of samples per millimetre) for the two imaging depths used in this study. Table 2 gives the number of scan lines and number of frames for each sampling setting. It also gives the spatial sampling frequencies in the azimuthal and elevational directions for two different axial depths, $7 \mathrm{~cm}$ and $15 \mathrm{~cm}$.

\section{$2.33 D$ displacement estimation algorithm}

The 2D displacement estimation program written in MATLAB (The Mathworks Inc., Natick MA, USA) and described in Hsu et al (2005) was rewritten to track in 3D. The

program estimates a $3 \mathrm{D}$ displacement vector $\hat{d}\left(d_{x}, d_{y}, d_{z}\right)$ at a point $P_{l}(r, \theta, \varphi)$ within a volume-image of the tissue phantom by performing a 3D cross-correlation search on the local speckle pattern. The program selects a reference volume, $A$, centred on point 
$P_{1}$, from a volume-image acquired at an initial start position. The $r, \theta, \varphi$ co-ordinates of $P_{1}$ were pre-programmed according to the position of the ultrasound field that was under investigation. A search volume, $B$, of equal size is selected from a user defined search region positioned in a second volume-image acquired after the transducer had been moved. The 3D normalised correlation coefficient, $\rho$, between $A$ and $B$ was calculated according to (Morsy and von Ramm 1998)

$\rho=\frac{\sum_{r=1}^{R} \sum_{\theta=1}^{L} \sum_{\varphi=1}^{F}\left(a_{r \theta \varphi}-\bar{a}\right)\left(b_{r \theta \varphi}-\bar{b}\right)}{\sum_{r=1}^{R} \sum_{\theta=1}^{L} \sum_{\varphi=1}^{F}\left(a_{r \theta \varphi}-\bar{a}\right)^{2} \sum_{r=1}^{R} \sum_{\theta=1}^{L} \sum_{\varphi=1}^{F}\left(b_{r \theta \varphi}-\bar{b}\right)^{2}}$

where $a$ is a voxel value within reference volume $A$ (containing $R$ rows, $L$ lines and $F$ frames), $b$ is a voxel value within search volume $B$ (of equal size), and $\bar{a}$ and $\bar{b}$ are the mean voxel values of volumes $A$ and $B$ respectively. $\rho$ was calculated for all possible positions of the search volume as it was successively shifted in increments of one voxel throughout the user defined search region. The size and position of the search region were chosen according to the magnitude and direction of the known displacement. The algorithm outputs a 3D matrix that describes the sampled crosscorrelation function $(c c f)$, which is the spatial distribution of $\rho$. The next step is to locate the sub-voxel position of the peak of the $c c f, P_{2}(r, \theta, \varphi)$, which indicates the most likely position that the reference volume has moved to. Peak localisation was performed by firstly finding the element within the correlation matrix with maximum $\rho\left(\rho_{\max }\right)$, giving the integer location of $P_{2}$. Sub-voxel location was then found using a 3-point 1D Gaussian fit to the correlation function, in all three dimensions. This was implemented using programs based upon code written for 3D particle tracking in 
particle imaging velocimetry called URAPIV (Gurka et al 1999). Finally, the Cartesian coordinates $P_{1}\left(x_{P 1}, y_{P 1}, z_{P 1}\right)$ and $P_{2}\left(x_{P 2}, y_{P 2}, z_{P 2}\right)$ were determined using equation 1 , and the displacement vector $\hat{d}$ found using

$$
\hat{d}=R^{-1} *\left(\left[\begin{array}{l}
x_{P 1} \\
y_{P 1} \\
z_{P 1}
\end{array}\right]-\left[\begin{array}{l}
x_{P 2} \\
y_{P 2} \\
z_{P 2}
\end{array}\right]\right)
$$

where $R$ is the rotation matrix between the coordinate system of the transducer and that of the translational stage (see Appendix A).

Displacement estimation was performed for at least 10 of the 20 pairs of volumes, giving rise to at least 10 displacement measurements. The first few volumes of each data acquisition (acquired in the first few seconds) were unusable, as artefacts were observed in the volume-images due to initial juddering of the probe when it began its sweeping motion. Switching the probe on a few seconds prior to motion tracking will avoid this problem for future application to organ tracking during therapy. The first usable volume in the start position was cross-correlated with the first usable volume in the finish position, the second usable volume in the start position was correlated with the second usable volume in the finish position, and so on.

\subsection{Parameters investigated}

As explained above, the main objectives of this study were to assess the feasibility of the technique and to investigate the tracking performance as a function of axial depth and scanner sampling setting. Other parameters that might be expected to influence 
the accuracy and precision of displacement estimation include the size of the reference volume used in tracking, the distance moved and direction of movement, and the azimuthal and elevational positions within the field of view. A complete list of the parameters investigated is shown in Table 3 . We expect that the larger the reference volume, the greater the measurement precision. However, for increased reference volume size, the spatial resolution of the technique decreases (Hsu et al 2005, Smith and Fenster 2000). We therefore investigated precision and accuracy as a function of reference volume size in order to determine the minimum size that would achieve adequate tracking. The number of distances investigated was restricted to three in order to limit the amount of data acquired. The choice of minimum and maximum distances was discussed above. A third distance of $2 \mathrm{~mm}$ was chosen because we are primarily concerned with tracking small distances. This is because when tracking organ motion during therapy, we wish to detect small movements out of the planning target volume, as this will improve treatment delivery accuracy.

\section{Results}

Accuracy and precision were investigated as a function of the parameters presented in Table 3. For one unique set of parameters, e.g. low sampling setting, $1 \mathrm{~mm}$ movement, $x$-direction, reference volume size $[9,9,9]$ and position $P_{l}$, the measured displacement is the mean tracked displacement averaged over 10 to 20 measurements. The standard deviation across these data is due to noise associated with the transducer, i.e., thermal noise, electronic amplifier noise and any possible error in the elevational position of the $1 \mathrm{D}$ transducer array (i.e., if the mechanical sweep is not perfectly reproducible). The standard deviation was found to be less than $0.05 \mathrm{~mm}$ for all measurements. Therefore, for results presented herein that were obtained using one 
unique set of parameters, the standard deviation is not presented. The error on the known displacements, i.e., the positional uncertainty associated with the translation stage, is $\pm 0.004 \mathrm{~mm}$ and therefore is also not considered further.

\subsection{Reference volume size, distance and direction of displacement}

The dependence of accuracy and precision on reference volume size (expressed as the number of voxels in one dimension, i.e., the number of rows, columns or frames) is shown in figures 2 to 4 for displacements in the $x, y$ and $z$ directions respectively. The mean displacement was obtained by averaging over a series of measured displacements with $P_{1}$ positioned at different depths on the central axis (between $4 \mathrm{~cm}$ and $13 \mathrm{~cm}$ in intervals of $0.8 \mathrm{~cm}$ ). The precision is defined as the standard deviation across this data set and therefore includes random variation due to different speckle patterns as well as any potential systematic variation with depth.

For $x$-displacements (figure 2), good accuracy (>95\%) and low standard deviation $(<3 \%)$ are obtained for all sampling settings, provided the size of the reference volume is at least $[7,7,7]$. In the $y$-direction (figure 3 ), very good accuracy and precision are obtained for all displacements, all sampling settings and all reference volume sizes with the exception of the smallest tracking volume $([3,3,3])$. Accuracy and precision are considerably poorer for $z$-displacements (figure 4). With high and medium sampling settings, good accuracy and precision are obtained for a reference volume size of at least $[11,11,11]$. For the low sampling setting, accuracies are poor for $1 \mathrm{~mm}$ and $2 \mathrm{~mm} z$-displacements; even for the largest reference volume size, the error is $\sim 0.5 \mathrm{~mm}$, corresponding to an accuracy of $50 \%$ and $75 \%$ for $1 \mathrm{~mm}$ and $2 \mathrm{~mm}$ 
distances respectively. From the above, it was decided that a reference volume of 5 rows, 7 columns and 11 rows $([5,7,11])$ would be used to obtain subsequent results.

For application to soft tissue tracking during therapy, we define adequate accuracy to be either $80 \%$ or an error of $0.5 \mathrm{~mm}$, whichever is lower (i.e., for $1 \mathrm{~mm}, 2 \mathrm{~mm}$ and $8 \mathrm{~mm}$ displacements, adequate accuracy means an error less than $0.2 \mathrm{~mm}, 0.4 \mathrm{~mm}$ and $0.5 \mathrm{~mm}$ respectively). Adequate precision is defined to be a standard deviation $\leq 5 \%$. Using these definitions and considering all data presented in figures 2 to 4 , it can be shown that $67 \%, 75 \%$ and $59 \%$ of $1 \mathrm{~mm}, 2 \mathrm{~mm}$ and $8 \mathrm{~mm}$ movements respectively were adequately tracked. This shows that tracking is poorest for $8 \mathrm{~mm}$ displacements. In some cases, the mean displacements differ from the known displacements by amounts greater than can be expected from the precision of the measurements. This is due to a systematic bias in the displacement measurement, which is discussed in section 4.6.

Figure 5 gives the mean measured displacement and precision (error bars) for the $x, y$ and $z$ directions for a volume tracked over an $x, y$ and $z$ movement $(x y z)$, where $d_{x}=d_{y}$ $=d_{z}$. Comparing the accuracy and precision in figure 5 with the results for pure motion $\left(d_{x}, d_{y}\right.$, and $\left.d_{z}\right)$ in figures 2,3 and 4 respectively, it is apparent that tracking performance has deteriorated for all displacement vector components.

\subsection{Correlation coefficient}

The peak of the correlation coefficient function, $\rho_{\max }$, is a measure of the degree of similarity between the reference volume before and after displacement. The greater the decorrelation, the lower the probability that the tracking algorithm can correctly identify the new position, meaning that $\rho_{\max }$ is an index of tracking precision (Morsy 
and Von Ramm, 1999). If this relationship can be confirmed for our probe, it suggests the potential for using $\rho_{\max }$ as a measure of the reliability of the tracked movement.

Mean precision (as defined above), for a reference volume of size [5, 7, 11], was calculated over all displacements $(x, y, z$, and $x y z, 1 \mathrm{~mm}, 2 \mathrm{~mm}$ and $8 \mathrm{~mm})$ and plotted as a function of $\rho_{\max }$ for the three different sampling settings (figure 6). Precision is plotted on a logarithmic scale. A separate logarithmic line of best fit was calculated for each sampling setting (figure 6). Pearson correlation coefficients between the data points and the best fit line were $0.63,0.84$ and 0.79 for high, medium and low sampling settings respectively, showing that there is a weak correlation between precision and $\rho_{\max }$. In figure 6 , the data points with standard deviation values of greater than $1 \mathrm{~mm}$ (i.e., poor precision) are for $8 \mathrm{~mm} z$-displacements imaged with low and medium sampling settings. Data points with the lowest standard deviation are for $y$-displacements. The considerable difference in the tracking performance for these two directions is the most likely explanation for the logarithmic trend in the data.

\subsection{Depth (axial position) dependence}

Depth dependence of tracking performance was assessed by positioning the reference volume at different axial positions along the central A-line of the volume image (i.e., for $P_{1}, \theta$ and $\varphi$ were kept constant at $0^{\circ}$ and $r$ was varied) for both $13 \mathrm{~cm}$ and $20 \mathrm{~cm}$ imaging depth data. Figures 7 to 9 give mean measured displacement, for $1 \mathrm{~mm}, 2 \mathrm{~mm}$ and $8 \mathrm{~mm}$ displacements respectively, as a function of depth. For $x y z$ motions, only the $z$-component of displacement is shown, denoted by $z(x y z)$, as this has the poorest accuracy and precision. In figure 9 , for the low sampling setting, the $z$ - and $z(x y z)-$

displacements are not included as they are not accurate (most exceed 15mm). Lines 
joining the $d_{z}$ data points have been drawn to aid the eye. Results for $y$-displacements are not given, as all were adequately tracked; the worst tracking performance was for a $2 \mathrm{~mm}$ movement at a depth of $18 \mathrm{~cm}$, for low sampling setting, where the accuracy was $95 \%$ (an error of $0.1 \mathrm{~mm}$ ) and the standard deviation was $0.5 \%$ (or $0.01 \mathrm{~mm}$ ).

We observe a decrease in accuracy as depth increases. This is more evident in the high and medium sampling data, as the low sampling accuracy is poor at all depths. For $x$-displacements, the decrease in accuracy with depth is likely to be due to an increase in the azimuthal beam width and an increase in spatial sampling. The decrease in accuracy with depth is even more apparent for the $z$ - and $z(x y z)$ displacements. This corresponds with the greater depth-dependent decrease in the elevational spatial resolution (relative to the azimuthal spatial resolution). In general, high sampling settings produce more accurate results than medium sampling at all depths and for all displacements. This indicates that increasing the spatial sampling at depth has improved the tracking performance, implying that a depth-dependent increase in elevational and azimuthal beam width cannot be the only cause of the degradation of tracking performance with depth. It is worth noting that the azimuthal focal depth is set automatically to a depth that is equal to the nearest integer depth in centimetres to the middle of the field of view. Hence, for these data, where the imaging depths were $13 \mathrm{~cm}$ and $20 \mathrm{~cm}$, the azimuthal focus occurred at 6 or $7 \mathrm{~cm}$ and $10 \mathrm{~cm}$ respectively. There was no obvious improvement in accuracy at these depths.

In summary, adequate tracking performance (denoted by the shaded areas in figures 7 to 9) was obtained for all $x$-displacements, using the high and medium sampling settings. Only the $8 \mathrm{~mm} x$-displacement was adequately tracked on the low sampling 
setting. Z-displacements were adequately tracked, using the high sampling setting, to depths of approximately $130 \mathrm{~mm}, 160 \mathrm{~mm}$ and $110 \mathrm{~mm}$ for $1 \mathrm{~mm}, 2 \mathrm{~mm}$ and $8 \mathrm{~mm}$ movements respectively. For $z(x y z)$ displacements, adequate tracking was obtained up to depths of $100 \mathrm{~mm}, 120 \mathrm{~mm}$ and $110 \mathrm{~mm}$ respectively.

Examination of the displacement tracking results reveals systematic depth-dependent trends in the $x$ - and $z$-displacement data. An example of this behaviour is seen in figure 8 , for the measurement of a $2 \mathrm{~mm} x$-displacement acquired using low sampling; the measured displacement shows an almost linear increase between $1.65 \mathrm{~mm}$ and $2.5 \mathrm{~mm}$ as depth increases. Investigation of the results has shown that is a direct result of bias in the sub-voxel displacement estimation.

\subsection{Azimuthal and elevational position}

Dependence of measured $x$-, $z$ - and $z(x y z)$-displacements on the azimuthal position $(\theta)$ and elevational position $(\varphi)$ of $P_{1}$ was investigated. Azimuthal and elevational sampling is symmetrical about the central axis and therefore we only investigated one half of the field. When varying the azimuthal position, the axial and elevational positions were fixed at $112.5 \mathrm{~mm}$ and $\varphi=0^{\circ}$ respectively and when varying the elevational position, the axial and azimuthal positions were fixed at $112.5 \mathrm{~mm}$ and $\theta$ $=0^{\circ}$ respectively. No dependence of tracking performance on the azimuthal or elevational position was observed.

\section{Discussion}

The goal of this study was to investigate the feasibility of the measurement of $3 \mathrm{D}$ displacements, in the absence of motion, using a commercially available curvilinear 
3D ultrasound probe. Firstly, we investigated the effect of the axial variation of spatial sampling and beam width on the accuracy and precision of tracked displacements and secondly, we assessed tracking performance as a function of the spatial sampling setting on the scanner. In this section, we discuss the results of these investigations and expand the discussion to show how the spatial sampling will affect the temporal resolution and hence, the maximum speed of organ motion that can be tracked.

\subsection{Direction of displacement}

Of the $x, y$ and $z$ directions, tracking performance was poorest in the $z$ direction. For $z$ movements, elevational motion is the major displacement component. Performance was expected to be worst in this direction because for 1D transducer arrays, the elevational spatial resolution is poorer than the azimuthal and axial spatial resolutions. The elevational direction also has the poorest spatial sampling frequency. However, if the ratio of sampling interval to beam width were the same in the elevational direction as in the azimuthal and axial directions, we would not necessarily expect poorer sampling to have any additional adverse effect on tracking performance.

Error in the elevational position of the tracked volume translates to an error in both $d_{y}$ and $d_{z}$. Using equation set 1 , it can be shown that for a depth of $10 \mathrm{~cm}$ and $\theta=0^{\circ}$, an error in the elevational position of $1^{\circ}$ will lead to an error of $2.2 \mathrm{~mm}$ in the $z$-direction and an error of $0.2 \mathrm{~mm}$ in the $y$-direction. This is particular to the curvilinear geometry of the probe and shows the importance of good tracking performance in all directions. 
Tracking accuracy and precision were poorer for $x y z$ movement than for $z$ movement. This is due to the additional decorrelation of the speckle pattern as the probe moves in all three dimensions.

\subsection{Distance moved}

Overall, the accuracy and precision was worst for $8 \mathrm{~mm}$ displacements. This is due to decorrelation of the speckle pattern. As the tracked volume moves away from the central axis, the angle at which the ultrasound beam is incident on the tracked volume changes and thus the speckle pattern also changes; for greater displacements, the angular change is larger. This effect is more pronounced at shallower depths because for a fixed Cartesian displacement, the azimuthal and/or elevational angular change is greater. Thus, when comparing the accuracy of $2 \mathrm{~mm}$ and $8 \mathrm{~mm}$ displacements in figures 8 and 9 , the poorer accuracy for $8 \mathrm{~mm}$ is more evident at shallower depths.

Angular decorrelation has implications for the maximum speed of organ motion that can be tracked, i.e., it is more probable that tissue can be tracked if it is displaced by $2 \mathrm{~mm}$ between consecutive volume acquisitions than if it is displaced by $8 \mathrm{~mm}$. Therefore, it may be necessary to ensure that the temporal resolution is sufficient to limit the movement of the tissue between acquisitions to just a few $\mathrm{mm}$. The temporal resolution of the probe is dependent on the spatial sampling and the size of the image volume; these relationships are discussed further in section 4.7. It is worth noting that even if angular decorrelation were not present, it would still be necessary to use a temporal resolution that restricts the inter-volume movement. This is because the purpose of motion tracking during therapy is to detect small movements of the organ before it moves out of the PTV (planning target volume). As we discussed previously 
(Hsu et al 2005), the principal motivation for developing methods of monitoring the inter- and intra-fraction organ position is to reduce the geometrical margin below the level currently used in radiotherapy of abdominal organs $(1-2 \mathrm{~cm})$. A reasonable aim is to reduce the margin (and hence the inter-volume movement) to a few mm.

\subsection{Sampling setting}

Tracking performance was better for the high sampling setting than it was for medium, and medium was better than low. There was poor accuracy for $z$ displacements using medium and low spatial sampling settings and therefore, at present, these settings are not useful for 3D tracking (see figures 7 to 9). However, a significant contributing factor to the poor accuracy at these lower spatial sampling settings is bias error in the sub-voxel displacement estimation (see section 4.6). Therefore, if this problem can be solved, tracking performance may improve for these settings. It should be noted that we also found that accuracy is adequate to track $x$ displacements using both high and medium sampling settings. Therefore, an optimum setting would combine the medium azimuthal sampling frequency with the high elevational sampling frequency, which would increase temporal resolution (relative to the high spatial sampling setting) whilst maintaining adequate tracking performance.

\subsection{Position}

The tracking accuracy was observed to decrease with depth, as was expected due to the increase in azimuthal and elevational beam width with depth beyond the azimuthal and elevational focal depths. The azimuthal focus occurred at depths of $\sim 7 \mathrm{~cm}$ and $10 \mathrm{~cm}$ for imaging depths of $13 \mathrm{~cm}$ and $20 \mathrm{~cm}$ respectively whilst the elevational focus occurred at $6 \mathrm{~cm}$. There was also a decrease in spatial sampling frequency with depth 
and it is difficult to determine which of these two factors was more important in the decline of tracking performance. However, at greater depths an improvement in tracking accuracy was seen when changing from medium and low sampling settings to high, implying that the two lower spatial sampling settings reduce tracking performance.

The rate of decrease in tracking accuracy with depth was displacement-dependent. It was worst for $8 \mathrm{~mm}$ (i.e., a more rapid decrease with depth was observed). As mentioned above, depending on the speed of organ motion, to maintain tracking performance it may be necessary to limit the distance moved between image volume acquisitions and therefore this will become increasingly necessary at greater depths.

The reference volume size used to investigate tracking performance as a function of depth $([5,7,11])$ was determined using data obtained at depths of $4 \mathrm{~cm}$ to $12 \mathrm{~cm}$. It is possible that, at greater depths, increasing the size of the reference matrix would increase tracking performance (Smith and Fenster 2000). For a linear transducer array with axially invariant sampling, if the size of the reference volume is fixed, then, as beam width increases as a function of depth, the amount of information contained in the reference volume will decrease and tracking performance will also decrease. However, for a curvilinear array, the spatial sampling frequency also decreases axially and therefore provided the rate of change of spatial sampling frequency is similar to the rate of increase in beam width, there will not be a significant reduction in the amount of information in the reference volume as a function of depth (i.e., in Cartesian space, the reference volume size increases with depth). Consequently, 
increasing the reference volume size at greater depths may not improve tracking performance.

The tracking performance did not vary as a function of azimuthal or elevational position. This was as expected because the spatial sampling and spatial resolution only vary as a function of axial position. If another mechanism for causing elevational and azimuthal dependence was present, it was too small to be observed. At the very azimuthal edges of the field, i.e., $\theta \sim 34^{\circ}$, we might see some change in accuracy and precision due to defocusing of the beam at these points (Goldstein 2000). However, due to the size of the search volume in the azimuthal direction ( 7 voxels), the midpoint of the reference volume did not approach such a high value of $\theta$.

\subsection{Correlation coefficient}

The precision of the displacement estimates decreased with decreasing $\rho_{\max }$ (figure 6). It is therefore possible that in the implementation of real-time $3 \mathrm{D}$ tracking, $\rho_{\max }$ could be used as a measure of the reliability of the tracked positions. For example, if $\rho_{\max }$ falls below a predetermined threshold, the displacement estimates could be "rejected" until the tracking system locates the tracked volume with greater precision. This is particularly important for any automated method of controlling patient treatment.

In this study, where the applied motion was translational, the main sources of decorrelation were electronic noise and change in the incident angle of the ultrasound beam. It has been shown for a linear array transducer that decorrelation of the speckle pattern would also occur due to tissue rotation and deformation (Meunier and Bertrand 1995). Although these have not been investigated in the present study, it is 
worth noting that rotation is in fact equivalent to a change in imaging angle. For example, an $8 \mathrm{~mm} x$-movement at an imaging depth of $4 \mathrm{~cm}$ produces a $5.7^{\circ}$ change in the angle of incidence of the ultrasound beam and this is adequately tracked (see figure 9). Typically, it is not expected that abdominal organs will rotate to a greater extent than $\sim 10^{\circ}$ (Aubry et al 2004) and therefore the amount of rotation could perhaps be restricted in a similar fashion to the amount of movement, i.e., by using a high temporal resolution. Note that this would also restrict the inter-volume speckle decorrelation due to tissue deformation.

The feasibility of tracking in the presence of rotation and deformation will be the subject of future work. The previous paragraph addressed the issue of how to deal with decorrelation caused by rotation and deformation. However, it may also be of interest to measure these types of motion, as this would provide more detailed information on the location of the target organ than a simple measure of global translation (Hsu et al, 2005). To measure non-translational motion, the reference volume would need to be successively moved to different parts of the ultrasound field to obtain the spatial distribution of displacement. However, this would increase the computation time and may make the procedure difficult to implement in real time.

\subsection{Error due to bias in the sub-voxel interpolation}

In some instances, a strong depth-dependent trend across all depths was observed in the measured displacement data (figures 7 to 9). This can be attributed to bias in the sub-voxel location of the peak of the cross-correlation function $(c c f)$. The magnitude of the bias error was greater for low and medium spatial sampling settings, which have larger sampling intervals. The bias error was also greater at greater depths where 
the sampling intervals are larger. The bias error is an absolute error and therefore it is more deleterious to the tracking accuracy of smaller displacements. For example, for the low sampling setting, a bias error of $0.4 \mathrm{~mm}$ was observed at a depth of $15 \mathrm{~cm}$. If we are tracking a $1 \mathrm{~mm}$ displacement, this introduces an inaccuracy of $40 \%$.

The Gaussian peak fit is based on the notion that the correlation peak has an approximately Gaussian shape; anything that does not approach this will lead to nonoptimal sub-pixel interpolation. This behaviour has been investigated by Nobach et al (2005), who demonstrate with the aid of simulated imaging functions, such as an airy function, that a non-Gaussian imaging function yields larger (up to a factor of 10) bias errors when using the Gaussian fit. From the results it is evident that, if we wish to track with medium and low sampling settings, the bias error will have to be reduced. Furthermore, as discussed above, we may wish to restrict the inter-volume movement and measure large (several centimetre) movements by summing up consecutive displacement estimates. In such a case, there is a theoretical possibility that the bias error would accumulate to produce a significant error on the total distance moved. Future work will investigate possible methods to reduce the bias error (Scarano 2002).

\subsection{Implications for temporal resolution}

As mentioned in sections 4.2 and 4.4, we may wish to restrict inter-volume movement, especially at greater depths, by increasing temporal resolution. The time it takes to acquire one ultrasound volume is

$t_{v o l}=L \times F \times 2 \times \frac{I D}{c}$ 
where $L$ is the number of lines per frame, $F$ is the number of frames, $I D$ is the imaging depth $(\mathrm{cm})$ and $c$ is the speed of sound $\left(\mathrm{cms}^{-1}\right)$. For a given imaging depth, the temporal resolution can be improved by reducing $L$ or $F$, i.e., by reducing the azimuthal sampling, elevational sampling, the azimuthal angle or the sweep angle. There are limitations on the extent to which these can be reduced. If sampling is too low, we will be unable to track with sufficient accuracy (section 4.3) and if the angles are too small, the field of view (FOV) will be reduced and the tissue will move out of the imaging volume during a treatment session. The maximum temporal resolution that can be achieved will determine the maximum organ speed that can be tracked. The following analysis uses the results of this study to examine maximum trackable organ speed as a function of depth.

We calculate the maximum trackable organ speed as a function of depth for two FOVs $(5 \mathrm{~cm}$ and $10 \mathrm{~cm})$, where FOV is defined as the size of the image matrix in both the $x$ and $z$ directions at the depth of the reference volume. These FOVs would be sufficient to track organ motion due to respiration, where the total distance moved is on the order of $3 \mathrm{~cm}$ (Balter et al 2001). The imaging angle needed to provide a particular FOV will decrease with depth. For example, for a $10 \mathrm{~cm} \mathrm{FOV,} \mathrm{at} \mathrm{a} \mathrm{depth} \mathrm{of}$ $10 \mathrm{~cm}$, we require azimuthal and sweep angles of $39^{\circ}$ and $44^{\circ}$ respectively, whereas at a depth of $15 \mathrm{~cm}$, we only require azimuthal and sweep angles of $29^{\circ}$ and $32^{\circ}$ respectively. The imaging angles will be reduced by a factor of two for the $5 \mathrm{~cm}$ FOV.

From the results discussed above, we identified that we require the high sampling setting to be able to accurately track $2 \mathrm{~mm}$ displacements (in the absence of object motion) in all directions to depths of $15 \mathrm{~cm}$. The high spatial sampling setting uses 
elevational sampling of $1^{\circ}$ per frame and azimuthal sampling of $0.56^{\circ}$ per line. We also showed that the medium sampling setting, which has azimuthal sampling of $0.66^{\circ}$ per line, is adequate to track $x$-displacements. As discussed above, it may be desirable to use a combination of "high" elevational sampling and "medium" azimuthal sampling. Therefore, the maximum trackable organ speed has been calculated for two situations, the high spatial sampling setting and a theoretical combination of the high and medium settings ("high/medium"). Assuming a $10 \mathrm{~cm}$ FOV and an imaging depth of $10 \mathrm{~cm}$, we will have 70 lines $(L=70)$ and 44 frames $(F$ $=44)$ for the high spatial sampling setting and 59 lines $(L=59)$ and 44 frames $(F=$ 44) for the high/medium spatial sampling setting. Using equation 4 , this results in $t_{v o l}$ being $0.4 \mathrm{~s}$ and $0.33 \mathrm{~s}$ for the high and high/medium sampling settings respectively. The maximum trackable organ speed is then given by the product of $1 / t_{v o l}$ and the maximum distance that can be tracked, which is assumed to be $2 \mathrm{~mm}$ (section 4.2 ).

Figure 10 gives the maximum trackable organ speed as a function of depth up to $15 \mathrm{~cm}$, which is the maximum depth at which $2 \mathrm{~mm} z$-displacements could be tracked. Previous studies have shown that as a result of respiratory motion, abdominal organs can move with speeds of up to $15 \mathrm{mms}^{-1}$ (Pernot et al 2004). From figure 10, we can see that using the high spatial sampling setting, it is feasible to track organs at these speeds, and at depths of 5 to $15 \mathrm{~cm}$, using a $5 \mathrm{~cm}$ FOV but not a $10 \mathrm{~cm}$ FOV. For a combination of high and medium sampling settings, which produces a $19 \%$ increase in maximum trackable organ speed, an organ speed of $15 \mathrm{mms}^{-1}$ can be tracked at all depths up to $15 \mathrm{~cm}$ using a $5 \mathrm{~cm}$ FOV. 
Considering that a small field of view may be required to increase temporal resolution, this suggests that the principal advantage of a curvilinear probe (its wide FOV) may not be relevant for this application. Therefore, a 3D linear probe might be more appropriate, especially as it would not be subject to the reduced tracking performance caused by angular decorrelation and poor spatial sampling at depth. However, commercially available 3D linear probes use high frequency transducers and would only be suitable for imaging superficial abdominal anatomy (up to $\sim 8 \mathrm{~cm}$ in depth).

Figure 10 is for one specific result. Further work may show this to be conservative, i.e., it may show that displacements greater than $2 \mathrm{~mm}$ (but less than $8 \mathrm{~mm}$ ) between volumes can be tracked. Furthermore, the spatial sampling requirements could be reduced if the bias error is reduced, further increasing temporal resolution. However, figure 10 is helpful in understanding the trade-offs between the maximum trackable organ speed, depth, and FOV and shows that as depth increases, the maximum trackable organ speed increases. This is due to the fact that for a fixed FOV in Cartesian space, the temporal resolution can be increased by reducing the azimuthal and elevational angles. This analysis has been based on results from measurements made using the "step and shoot" method of acquisition and therefore does not include any potential reduction in the maximum trackable organ speed due to motion during volume acquisition. This will be the subject of future work.

\subsection{Real-time implementation}

The displacement calculations reported in this study were computed off-line. The eventual goal is to perform 3D displacement estimation in real-time. As discussed 
previously (Hsu et al 2005), real-time 2D elastography, which requires a 2D crosscorrelation search at many different locations in the $2 \mathrm{D}$ ultrasound image, is commercially available. Our cross-correlation is performed in 3D but is for one location only, therefore the number of computations is comparable and it is expected that real-time implementation is feasible. Optimisation schemes to reduce the computational cost of 3D correlation based speckle tracking and a hardware solution based on FPGAs are discussed in Chen et al (2005).

\section{Conclusions}

This study has shown that a commercially available 3D curvilinear probe can be used to track 3D speckle patterns that undergo pure $x, y, z$ and $x y z$ motions. Accuracy and precision are adequate to track small displacements $(1 \mathrm{~mm}$ to $2 \mathrm{~mm})$ for application to motion tracking in therapy using the scanner's "high" sampling setting. Correlation coefficient was shown to decrease with precision and therefore may have the potential to be used as a measure of confidence in the tracking system. We have shown that larger displacements result in greater decorrelation of the speckle pattern due to angular change. Therefore, we require sufficient temporal resolution to limit the distance moved between volume acquisitions. Given the observation of good tracking performance for $2 \mathrm{~mm}$ displacements with the high sampling setting, we have estimated maximum trackable organ speeds as a function of depth.

\section{References}

Aubry et al 2004 Measurements of intrafraction motion and intrafraction rotation of prostate by three-dimensional analysis of daily portal imaging with radiopaque markers Int. J. Radiat. Oncol. Biol. Phys. 60 30-39 
Bouchet LG et al 2000 Calibration of three-dimensional ultrasound images for imageguided radiation therapy Med. Phys. Biol. 46 559-577

Balter J et al 1995 Automated localisation of the prostate at the time of treatment using implanted radiopaque markers Int. J. Radiat. Oncol. Biol. Phys. 38 1281-1286

Balter JM et al 2001 Determination of ventilatory liver movement via radiographic evaluation of diaphragm position Int. J. Radiat. Oncol. Biol. Phys. 51 267-270

Chandra A 2003 Experience of ultrasound-based daily prostate localisation Int. J. Radiat. Oncol. Biol. Phys. 56 436-477

Chen et al 2005 3D correlation-based speckle tracking Ultrson. Imaging 20 151-159

Cury FLB et al 2006 Ultrasound-based image guided radiotherapy for prostate cancer

- Comparison of cross-modality and intramodality methods for daily localization during external beam radiotherapy Int. J. Radiat. Oncol. Biol. Phys. 56 436-477

Fenster A, Downy DB and Cardinal 2001 Three-dimensional ultrasound imaging Med. Phys. Biol. 49 R67-R99

Goldstein A 2000 The effect of acoustic velocity on phantom measurements Ultra. Med. Biol. 26 1133-1143

Gurka R et al 1999 Computation of pressure distribution using PIV velocity data Proc. 3rd Int. Workshop on PIV'99 - Santa Barbara California USA 16-18 Sept.671676

Hsu A et al 2005 Feasibility of using ultrasound for real-time tracking during radiotherapy Med. Phys. 32 1500-1512 
Keall PJ et al 2004 On the use of EPID-based implanted marker tracking for 4D radiotherapy Med. Phys. 31 3492-3499

Meunier J and Bertrand M 1995 Ultrasonic texture motion analysis: Theory and simulation IEEE Trans. Ultrason. Ferroelectr. Freq. Contr. 14 293-300

Morsy AA and von Ramm OT 1998 3D ultrasound tissue motion tracking using correlation search Ultrasonic Imaging 20 151-159

Morsy AA and von Ramm OT 1999 FLASH Correlation: A new method for 3D ultrasound tissue motion tracking and blood velocity estimation IEEE Trans. Ultrason. Ferroelectr. Freq. Contr. 46 729-735

Nederveen AJ et al 2002 Measurements and clinical consequences of prostate motion during a radiotherapy fraction Int. J. Radiat. Oncol. Biol. Phys. 53 206-214

Nioutsikou E et al 2006 Quantifying the effect of respiratory motion on lung tumour dosimetry with the aid of a breathing phantom with deforming lungs Med. Phys.

Biol. 51 3359-3374

Nobach H et al 2005 High-precision sub-pixel interpolation in particle image velocimetry image processing Experiments in fluids 39 299-304

Pernot M, Tanter M and Fink M 2004 3-D real-time motion correction in highintensity focussed ultrasound therapy Ultra. Med. Biol. 30 1239-1249

Scarano F 2002 Iterative image deformation methods in PIV Meas. Sci. Technol. 13 R1-R19 
Shimizu S et al 2000 Use of an implanted marker and real-time tracking of the marker for the positioning of prostate and bladder cancers Int. J. Radiat. Oncol. Biol. Phys. 48 $1591-1597$

Smith WL and Fenster A 2000 Optimum scan spacing for three-dimensional ultrasound by speckle statistics Ultra. Med. Biol. 26 551-562

Smith SW, Pavy H G Jr and von Ramm O T 1991 High-speed ultrasound volumetric imaging system. Part I. Transducer design and beam steering IEEE Trans. Ultrason. Ferroelectr. Freq. Contr. 38 100-8

Webb S 2006 Motion effects in intensity modulated) radiation therapy: a review Med. Phys. Biol. 51 R403-R425

\section{Appendix A}

The transformation between the coordinates of a point in the ultrasound frame of reference, $M_{U}$, and the coordinates of the same point in the frame of reference of the translational stage, $M_{S}$, is given by:

$$
M_{U}=R M_{s}+T
$$

where $R$ is a $3 \times 3$ rotation matrix and $T$ is a $3 \times 1$ translation matrix. Equation A1 can be solved by finding the coordinates of a set of three non-coplanar points in both frames of reference. The use of more than three points increases the precision with which $R$ and $T$ can be determined. Using $N$ points, such that the matrices $M_{U}$ and $M_{S}$ each contain $3 \times N$ elements, the rotation and translation matrices are obtained by minimising $\varepsilon^{2}$ in the following least-square fit equation (Bouchet et al. 2001):

$\mathcal{E}^{2}=\sum_{n=1}^{N}\left\|M_{U, n}-\left(R M_{S, n}+T\right)\right\|^{2}$ 
The mutual points were obtained by embedding a $0.4 \mathrm{~mm}$-diameter ball-bearing in a gelatine phantom and acquiring volume-images of the ball-bearing at 50 different transducer positions $(N=50)$.

Or aim was to compare measured and known displacements. The displacement between two points depends only on their relative (not absolute) positions, so the translation matrix, $T$, did not need to be determined. If all points are transformed such that they are referred to the centroids of the sets of points in ultrasound and stage space respectively (the transformed coordinates being denoted by $M_{U C}$ and $M_{S C}$ ), $R$ can be determined independently of $T$, by minimising (Bouchet et al 2001):

$\mathcal{E}_{R}^{2}=\sum_{n=1}^{N}\left\|M_{U C, n}-R M_{S C, n}\right\|^{2}$

Equation A3 was solved in MATLAB, using singular value decomposition, with the

line of code $[U, W, V]=\operatorname{svd}\left(M_{s c} * M_{U c}^{\prime}\right)$, where the superscript ${ }^{\prime}$ denotes the matrix transpose. The rotation matrix was then given by the line of code $R=V^{*} U^{\prime}$.

\section{Acknowledgements:}

The authors would like to acknowledge Paul Barbone and Michael Scott Richards of the University of Boston for their modifications to the PIV tracking code and Cancer Research-UK for funding this work under Programme Grant Reference No.

C46/A3970. 


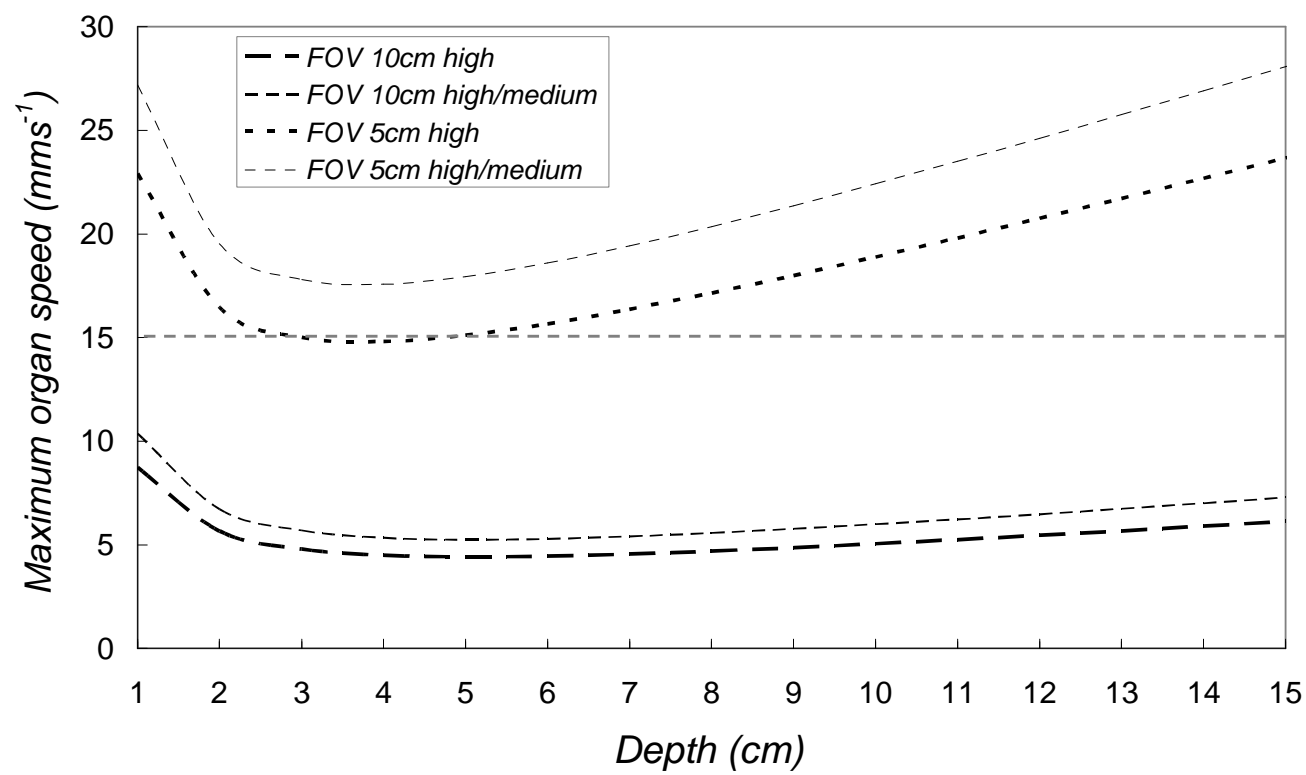

Figure 10. Maximum trackable organ speed as a function of depth for $5 \mathrm{~cm}$ field of view and a $10 \mathrm{~cm}$ field of view and using the high and high/medium sampling settings. 


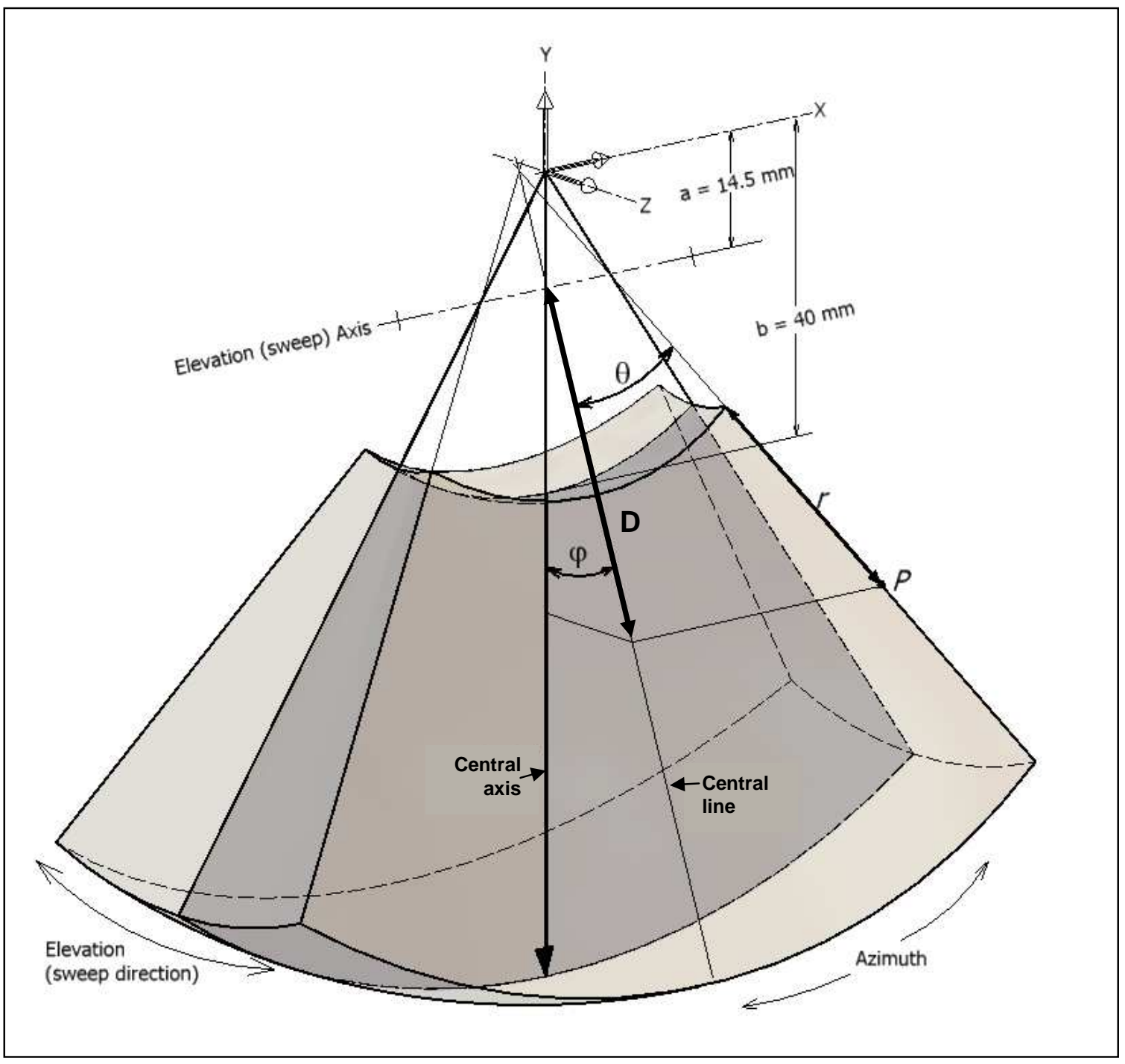

Figure 1. Schematic diagram of the imaging geometry. $x, y$ and $z$ directions (translation stage motions) are shown relative to elevational, azimuthal and axial directions. Distances $a$ and $b$ are $14.5 \mathrm{~mm}$ and $40 \mathrm{~mm}$ respectively, where $b$ is the radius of curvature of the transducer. The central axis is coincidental with the $y$-axis. 


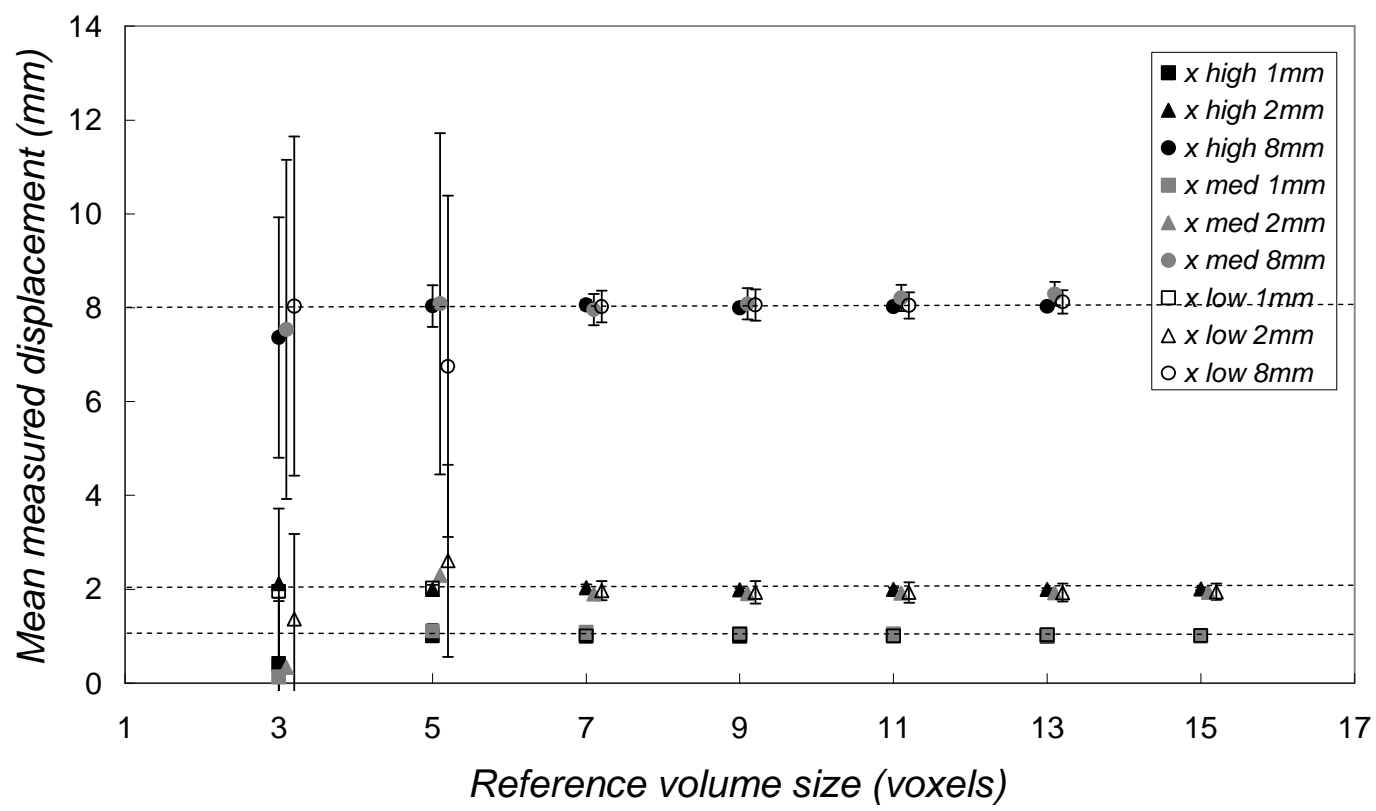

Figure 2. Mean measured displacement and mean precision (error bars) as a function of reference volume size (expressed as the number of voxels in one dimension) and spatial sampling setting, for 1,2 , and $8 \mathrm{~mm}$ displacements in the $x$ direction. Data points have been staggered along the horizontal ordinate to separate the error bars.

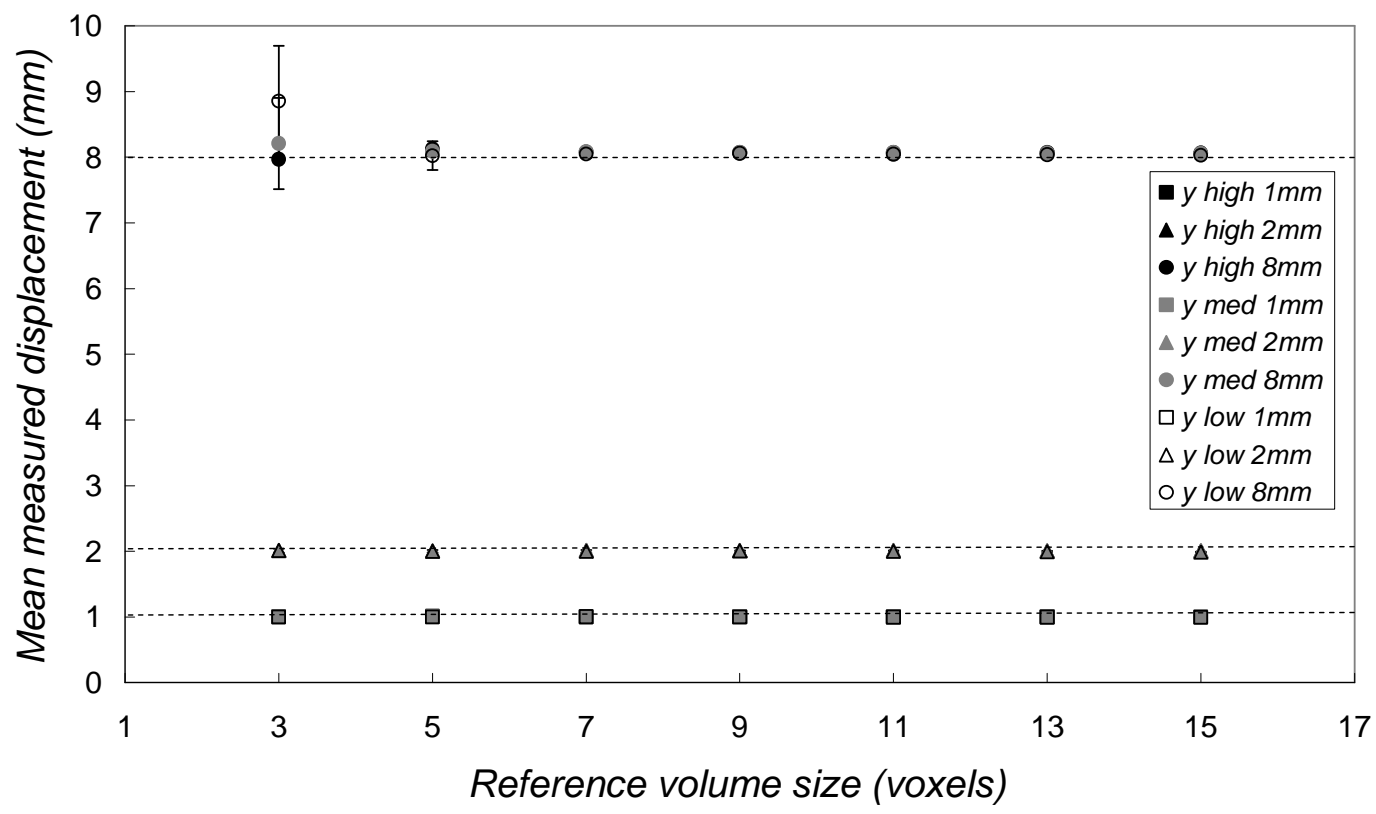

Figure 3. Mean measured displacement and mean precision (error bars) as a function of reference volume size (expressed as the number of voxels in one dimension) and spatial sampling setting, for 1,2 , and $8 \mathrm{~mm}$ displacements in the $y$ direction. Data points have been staggered along the horizontal ordinate to separate the error bars. 


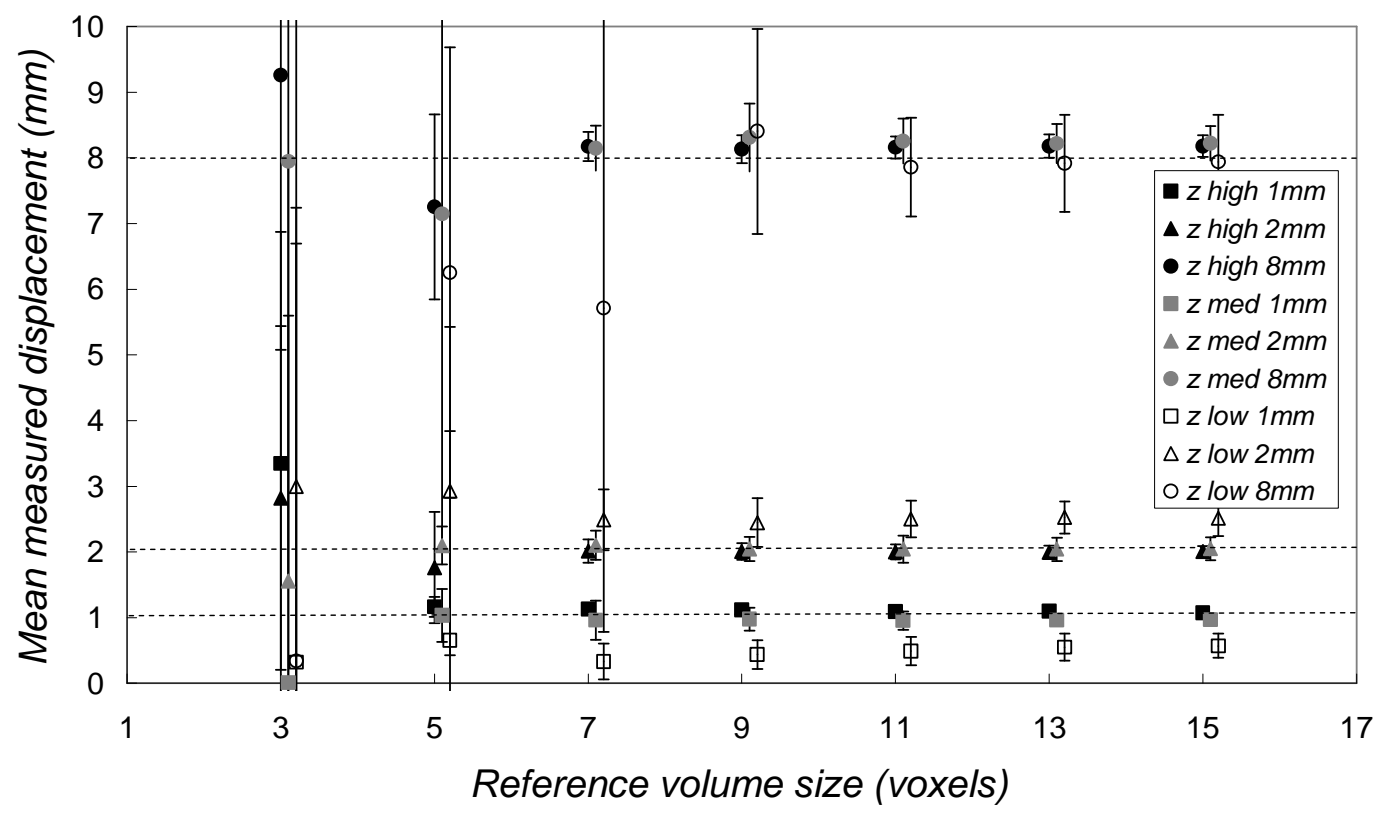

Figure 4. Mean measured displacement and mean precision (error bars) as a function of reference volume size (expressed as the number of voxels in one dimension) and spatial sampling setting, for 1,2 , and $8 \mathrm{~mm}$ displacements in the $z$ direction. Data points have been staggered along the horizontal ordinate to separate the error bars.

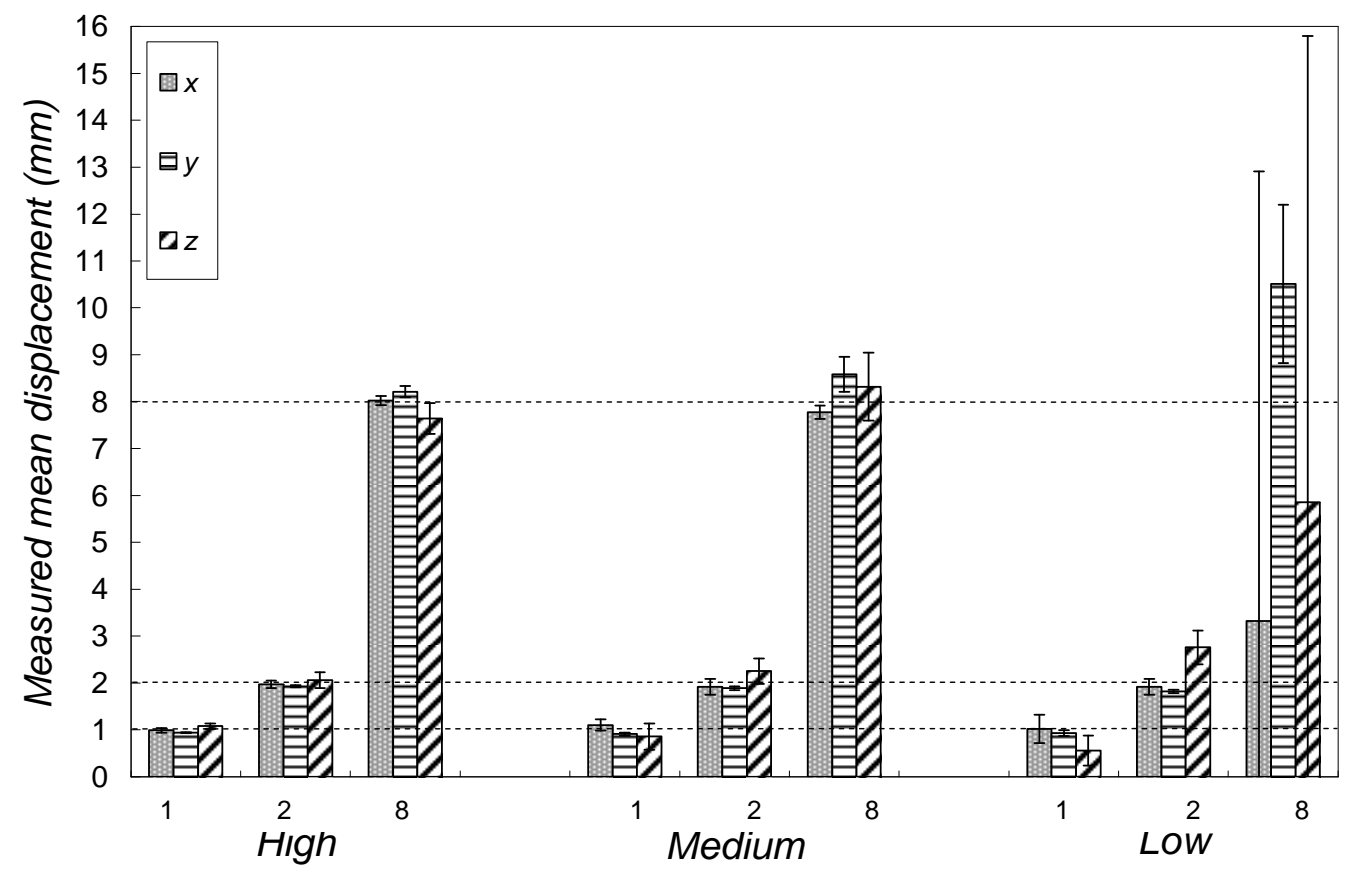


Figure 5. Mean measured displacement in $x, y$ and $z$ directions for $x y z$ displacements averaged over displacements measured at $8 \mathrm{~mm}$ intervals between depths of $4 \mathrm{~cm}$ and $13 \mathrm{~cm}$ at the central axis for the high, medium and low spatial sampling setting using reference volume size $[5,7,11]$.

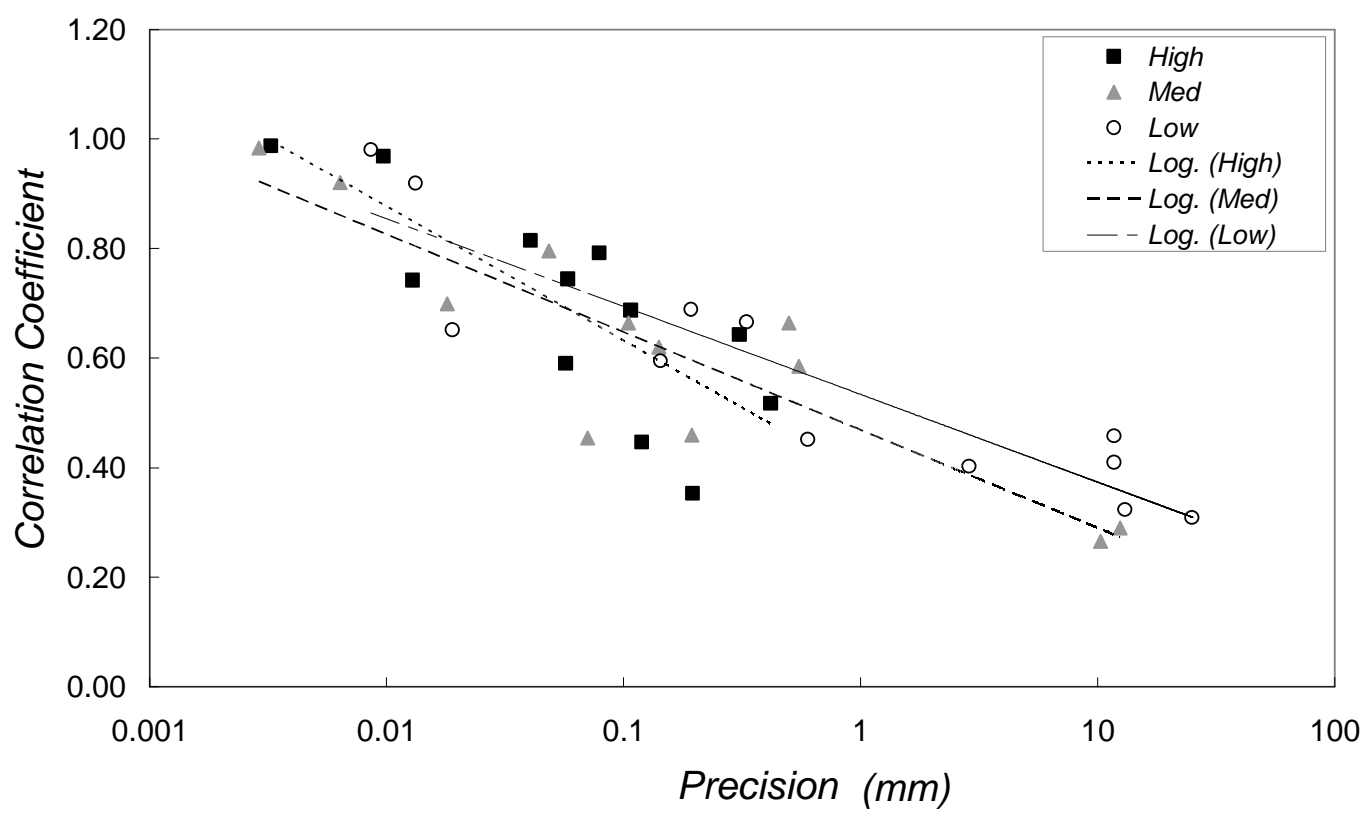

Figure 6. Correlation coefficient as a function of precision for 1,2 and $8 \mathrm{~mm}$ displacements, $x, y, z$ and $z(x y z)$ motions. Logarithmic trend lines have been fitted to high, medium and low data sets with correlation coefficients of $0.63,0.84$ and 0.79 respectively. 


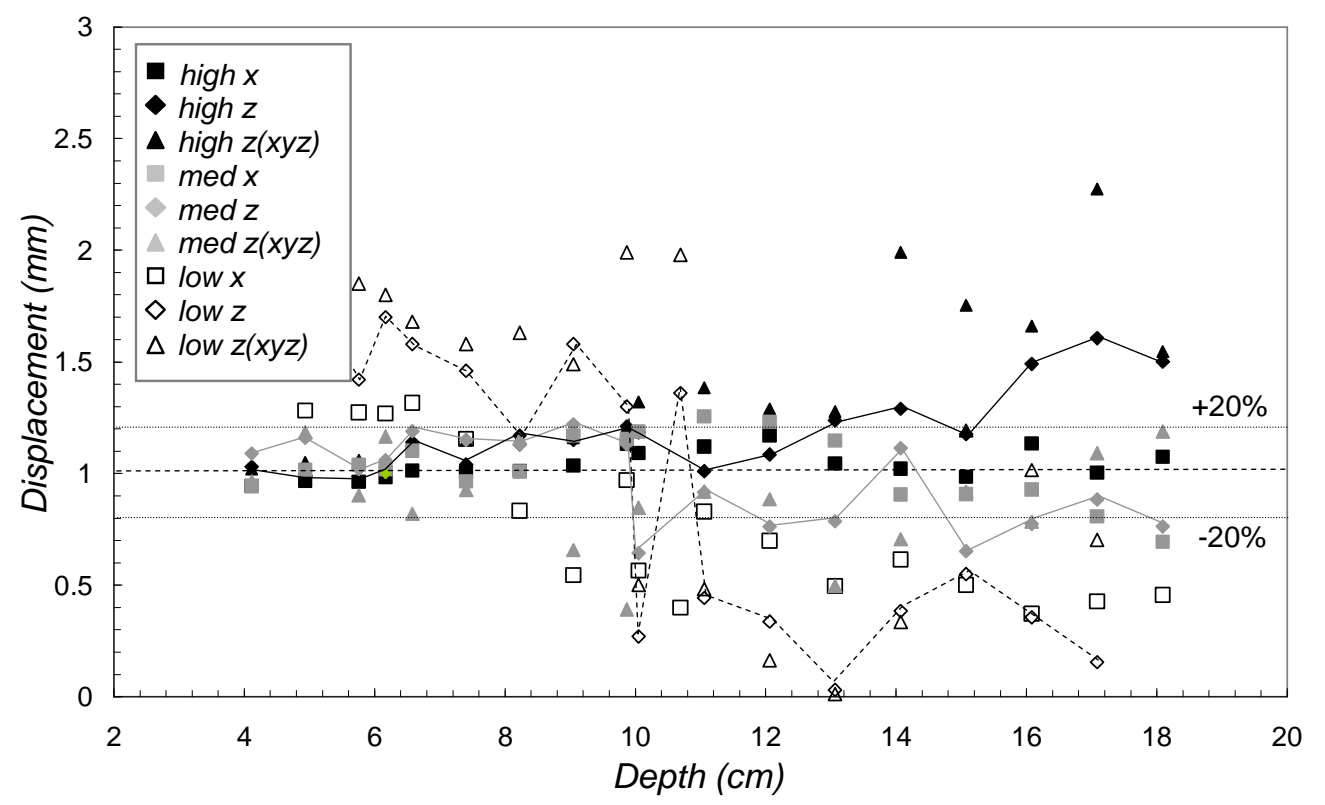

Figure 7. Measured displacement as a function of reference volume depth for $1 \mathrm{~mm} x$, $z$ and $z(x y z)$ displacements for the high, medium and low spatial sampling settings using reference volume size $[5,7,11]$.

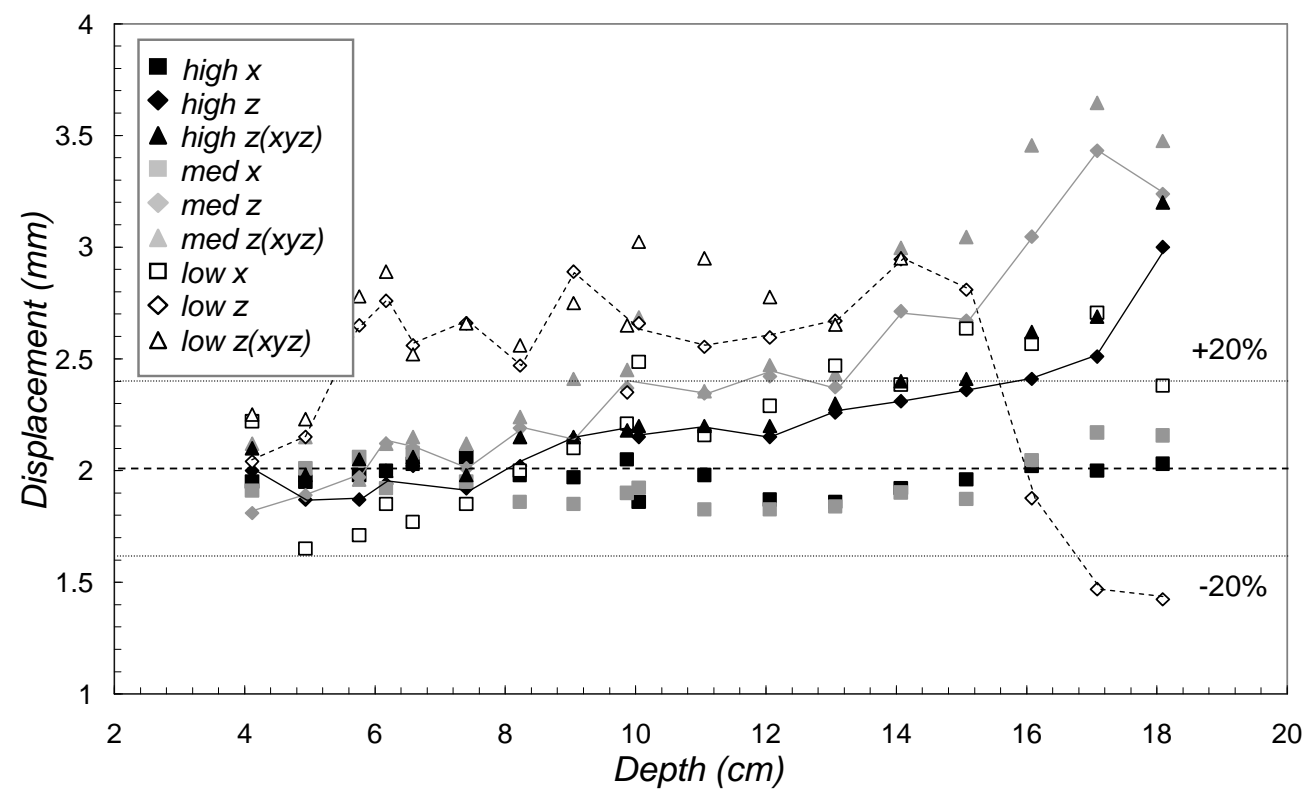

Figure 8. Measured displacement as a function of reference volume depth for $2 \mathrm{~mm} x$, $z$ and $z(x y z)$ displacements for the high, medium and low spatial sampling settings using reference volume size $[5,7,11]$. 


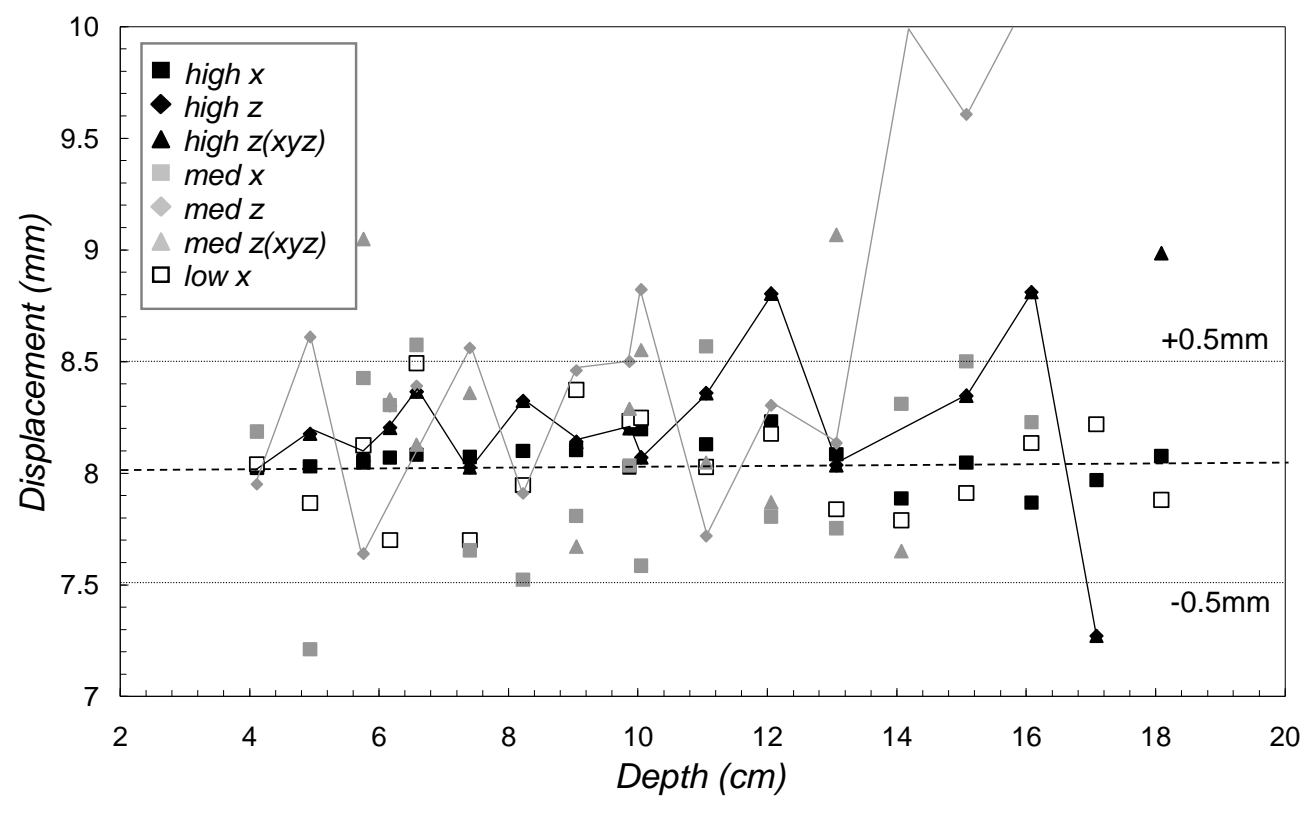

Figure 9. Measured displacement as a function of reference volume depth for $8 \mathrm{~mm} x$, $z$ and $z(x y z)$ displacements for the high, medium and low spatial sampling settings using reference volume size $[5,7,11]$. 